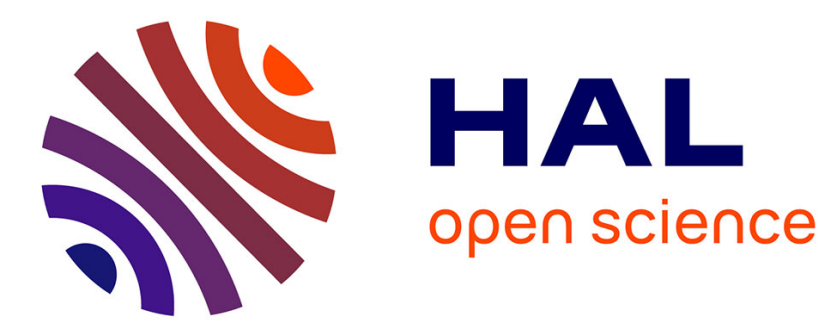

\title{
Multiple-Star System Adaptive Vortex Coronagraphy Using a Liquid Crystal Light Valve
}

\author{
Artur Aleksanyan, Nina Kravets, Etienne Brasselet
}

\section{To cite this version:}

Artur Aleksanyan, Nina Kravets, Etienne Brasselet. Multiple-Star System Adaptive Vortex Coronagraphy Using a Liquid Crystal Light Valve. Physical Review Letters, 2017, 118 (20), pp.203902. 10.1103/PhysRevLett.118.203902 . hal-01526070

\section{HAL Id: hal-01526070 \\ https://hal.science/hal-01526070}

Submitted on 22 May 2017

HAL is a multi-disciplinary open access archive for the deposit and dissemination of scientific research documents, whether they are published or not. The documents may come from teaching and research institutions in France or abroad, or from public or private research centers.
L'archive ouverte pluridisciplinaire HAL, est destinée au dépôt et à la diffusion de documents scientifiques de niveau recherche, publiés ou non, émanant des établissements d'enseignement et de recherche français ou étrangers, des laboratoires publics ou privés.

\section{다(1)(2)}

Distributed under a Creative Commons Attribution - ShareAlikel 4.0 International 


\title{
Multiple-Star System Adaptive Vortex Coronagraphy Using a Liquid Crystal Light Valve
}

\author{
Artur Aleksanyan, Nina Kravets, and Etienne Brasselet ${ }^{*}$ \\ Université Bordeaux, CNRS, LOMA, UMR 5798, F 33400 Talence, France
}

\begin{abstract}
We propose the development of a high contrast imaging technique enabling the simultaneous and selective nulling of several light sources. This is done by realizing a reconfigurable multiple vortex phase mask made of a liquid crystal thin film on which local topological features can be addressed electro optically. The method is illustrated by reporting on a triple star optical vortex coronagraphy laboratory demonstration, which can be easily extended to higher multiplicity. These results allow considering the direct observation and analysis of worlds with multiple suns and more complex extrasolar planetary systems.
\end{abstract}

Two decades after the first indirect detection of extrasolar planets (i.e., exoplanets) around a Sun-like star [1] by Doppler velocimetry, astronomers are nowadays equipped with a large set of techniques enabling the identification of planets and planetary systems [2]. The current observing campaign of the second mission of the Kepler space telescope is one example of the tools that have been designed to discover Earth-like planets hosted in the socalled habitable zone [3]. Among the various methods to find a planet [4] such as Doppler velocimetry, transit, gravitational microlensing, timing, or astrometry, the detection of light from a planet itself via direct imaging is a prime choice since it provides detailed information about its surface or its atmosphere. The first direct image of an exoplanet was reported more than one decade ago [5] from terrestrial observations at the Very Large Telescope, at the same time when the concept of so-called vortex coronagraphy emerged [6,7], roughly 70 years after Lyot invented the coronagraph in the 1930s in the framework of his studies on the corona of the Sun.

A coronagraph is an instrument that provides selective starlight rejection, hence, allowing the direct observation of fainter nearby astronomical objects. The original idea of Lyot was to selectively occult sunlight by placing an opaque disk in the focal plane of a telescope followed by a pupil diaphragm [8]. Since then, several coronagraphs have been developed and installed in terrestrial and space telescopes. In particular, the first direct image of an exoplanet by a Lyot coronagraph was taken by the Hubble Space Telescope in 2008 [9], while the first exoplanetary image from a vortex coronagraph was reported in 2010 using the Hale Telescope [10]. The kind of coronagraph of interest in the present study is the vortex coronagraph, which relies on a phase mask imparting a spiraling phase profile to the starlight. Ideally, such a mask has a complex amplitude transmission function of the form $\tau_{\text {ideal }}(\phi)=\exp (i \ell \phi)$ where $\ell$ is an even integer and $\phi$ is the azimuthal angle in the focal plane. By placing such an ideal vortex mask in the focal plane of a telescope with a uniformly illuminated circular-shaped entrance pupil and centering it on a starlight intensity pattern, one gets peripheral redistribution of the latter outside an area of null intensity in the exit pupil plane. Therefore, starlight can be suppressed by merely placing an iris in the exit pupil plane, while the off-axis signal is almost unaffected for angular separation larger than the diffraction limit [11]. Although vortex coronagraphy emerged only one decade ago [6,7], this technique now equips all of the main telescopes (e.g., Hale, Keck, Large Binocular Telescope, Subaru, and Very Large Telescope) [12] and its development thus represents great technological interest.

Now that more than 3500 planets have been confirmed, among which around 600 are multiple-planet systems [13], optical vortex coronagraphy has a bright future. However, a vortex coronagraph can reject one star at a time while the fraction of multiple-star systems in the Milky Way is not yet precisely ascertained but is expected to be nonnegligible. This implies a shortcoming in terms of the number of exoplanets that could be imaged in a direct manner since the detection of worlds with two suns [14] and more complex planetary systems [15] started a few years ago. In this context, a coronagraph enabling the simultaneous and selective nulling of multiple-star systems would be desirable. To date, only a few attempts have been made to null multiple-star systems, in particular, binary stars. One can mention the on-sky demonstration at the Palomar Observatory [16] using a linear band-limited coronagraphic mask, which is, however, restricted to one-dimensional obscuration of faint companions along the mask axis. A dual-mask apodized pupil Lyot coronagaph was also proposed [17] as well as multistar wavefront control [18]. Still, previous studies are all limited to the case of binary stars and the assets of optical vortex coronagraphy have not yet been implemented in that context.

Here we address multiple-star system optical vortex coronagraphy. For this purpose, liquid crystals (LCs) are interesting candidates since efficient field-induced optical vortex masks with $|\ell|=2$ can be realized from LC 
topological defects with strength $s= \pm 1$ (i.e., the optical axis winds by $\pm 2 \pi$ over a full turn around the defect core). Several demonstrations of LC optical vortex masks have been reported in recent years, e.g., via purely optical [19], purely electrical [20], optoelectrical [21,22], and optothermal [23] strategies. Remarkably, all these examples correspond to self-engineered vectorial vortex masks whose potential in vortex coronagraphy has been recently discussed [24]. Although promising, the latter demonstration is, however, hardly generalizable to create positioncontrolled multiple-vortex masks. Indeed, the obtained material vortices are randomly distributed and may be distorted and unsteady. In contrast, by using a liquid crystal light valve (LCLV) [25], here we report on the optical vortex coronagraphy laboratory demonstration of a simulated triple-star system, whose extension to higher multiplicity is straightforward.

The LCLV consists of a $L=13$ - $\mu$ m-thick nematic LC layer (MLC6608, from Merck, characterized by a negative relative dielectric permittivity $\epsilon_{a} \simeq-4.2$ at $1 \mathrm{kHz}$ frequency and $20^{\circ} \mathrm{C}$ temperature) sandwiched between a 1-mm-thick slab of bismuth silicon oxide (BSO) photoconductive crystal and a 1-mm-thick glass slab, which are both of which have transparent electrodes, see Fig. 1(a). The inner walls of the cell are treated with a surfactant (cethyltrimethyl-ammonium-bromide) that provides uniform LC orientation along the normal to the slab without applied external field. According to Ref. [21], a localized umbilic with $s=1$ can be generated in the presence of an applied voltage by a light beam owing to the photorefractive properties of BSO. As shown in Fig. 1(a), we use a circular-shaped uniform writing illumination with diameter
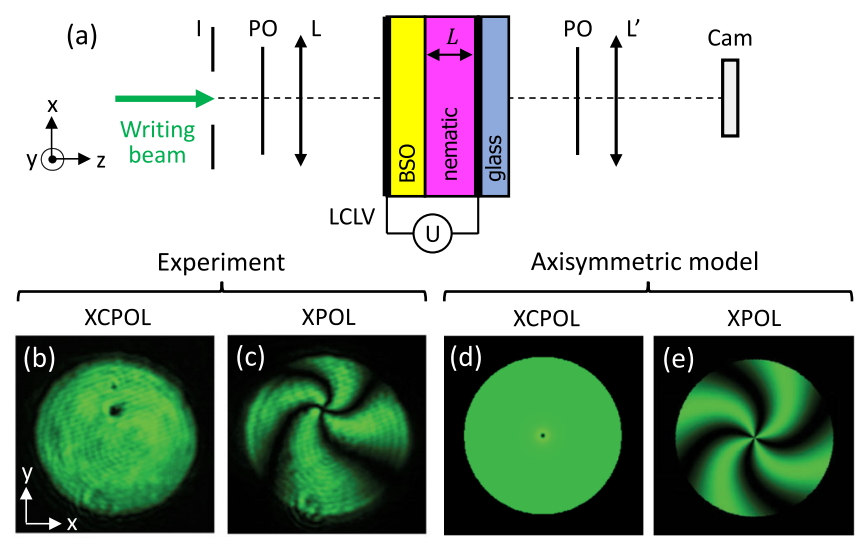

FIG. 1. (a) Localized vortex mask setup. $I$, iris; PO, polarization optics; $L$ and $L^{\prime}$, lenses; LCLV, liquid crystal light valve; Cam, camera; $U$, quasistatic applied voltage. (b) Image under crossed circular polarizers (XCPOL) of the sample illuminated by a uniformly illuminated circular shaped mask beam at $532 \mathrm{~nm}$ wavelength. (c) Same as panel (b) but in the case of crossed linear polarizers (XPOL). Panels (d) and (e) are the simulated counter parts of panels (b) and (c) assuming an axisymmetric retardance profile.
$D \simeq 250 \mu \mathrm{m}$ from a laser at wavelength $\lambda_{W}=532 \mathrm{~nm}$ and total power $P_{W} \simeq 0.36 \mathrm{~mW}$. This is made by imaging a $2 \mathrm{~mm}$ diameter iris $(I)$ on the LC layer with a lens $(L)$, while the applied voltage is set at $U \simeq 11.1 V_{\mathrm{rms}} \simeq 0.9 U_{\text {th }}$ where $U_{\text {th }}$ refers to the threshold value below which the whole LC layer remains at rest in the absence of light. The obtained localized vortex mask placed between crossed circular (linear) polarizers [XCPOL (XPOL)] is then imaged on a camera (Cam) by another lens $\left(L^{\prime}\right)$ as shown in Figs. 1(b) and 1(c). The dark spot in the XCPOL image is reminiscent of the nonsingular core of an umbilical defect that is associated with zero LC reorientation, while the four dark brushes in the XPOL are the signature of an umbilic with unit strength. We notice that the observed spontaneous broken axisymmetry [i.e., off-axis defect core see Fig. 1(b)] and chiral broken symmetry [i.e., swirled in-plane reorientation pattern, see Fig. 1(c)] are general features of LCLVs already mentioned in earlier works [21,26].

Anticipating later simulations, we propose a simplified, yet robust attempt to describe the created vortex mask by characterizing the inhomogeneous anisotropic slab by an axisymmetric birefringent phase retardation $\Delta(r)$, and a swirled distribution for the optical axis orientation angle $\psi(r, \phi)$. In experiment, the settings are chosen to maximize XCPOL transmission of an incident circularly polarized "star" light beam (at $633 \mathrm{~nm}$ wavelength) centered on an umbilic. Accordingly, the optoelectrically written umbilic is described by a mask having a piecewise retardance profile of the form $\Delta(r)=\Delta_{\infty} a^{2}\left(r / r_{c}\right)$ for $r<D / 2$ and zero otherwise, where $a(\rho)$ is a monotonous function derived by Rapini satisfying the partial differential equation $d^{2} a / d \rho^{2}+(1 / \rho) d a / d \rho+\left(1-1 / \rho^{2}\right) a-a^{3}=0 \quad$ where $a(0)=0$ and $a(\infty)=1[27,28]$ and $r_{c}$ is the external field-dependent core radius. Using the XCPOL intensity expression $I_{\mathrm{XCPOL}}(r) \propto \sin ^{2}[\Delta(r) / 2]$, maximal XCPOL transmission at $633 \mathrm{~nm}$ is found for $\Delta_{\infty} \simeq 1.1 \pi$ and $r_{c} \simeq 4 \mu \mathrm{m}$. On the other hand, we choose a Gaussian swirl ansatz for the optical axis profile, namely, $\psi(r, \phi)=\phi+\psi_{0} \exp \left(-2 r^{2} / w_{\psi}^{2}\right)$, with $\psi_{0} \simeq-1.1$ and $w_{\psi} \simeq 140 \mu \mathrm{m}$, which gives a XPOL intensity pattern of the form $I_{\mathrm{XPOL}}(r, \phi) \propto \sin ^{2}[2 \psi(r, \phi)]$. The latter description of the localized umbilic is compared with experiments in Fig. 1.

Then, we show that the obtained localized optical vortex mask has some potential in terms of optical vortex coronagraphy despite the exhibited spontaneous broken axial and chiral symmetries. The demonstration is made by using the setup shown in Fig. 2 that is a typical setup for the laboratory demonstration of a coronagraph. At first, the coronagraphic capabilities of the LCLV, which is placed in the focal plane of the telescope, is tested by using a single on-axis writing beam $\left(W_{1}\right)$. The disk-shaped illumination from the beam $W_{1}$ is adjusted in order to obtain an overlap between the core of the generated LC defect and the Airy spot obtained from a quasiplane wave laser beam 


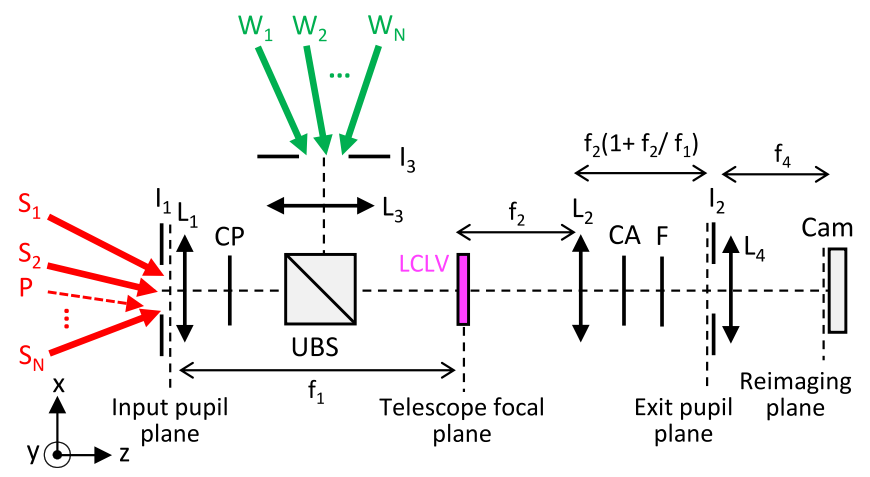

FIG. 2. Multiple star vortex coronagraph laboratory demon stration setup. $W_{i}$, writing beams generating on demand localized vortex masks; $S_{i}$, starlight beams mimicking pointlike sources; $I_{i}$, irises; $L_{i}$, lenses with focal length $f_{i} ; C P, \mathrm{CA}$, circular polarizer and analyzer; UBS, unpolarized beamsplitter; $F$, Notch filter at 532 nm; Cam, camera. Light sources are mutually incoherent.

at wavelength $\lambda_{S}=633 \mathrm{~nm}$ that mimics starlight $\left(S_{1}\right)$. Starlight $S_{1}$ is clipped by the input pupil of the telescope (lenses $L_{1}$ and $L_{2}$ of focal length $f_{1}=f_{2}=200 \mathrm{~mm}$ ) defined by the circular iris $I_{1}$ of radius $R_{1}=2 \mathrm{~mm}$, which gives an Airy spot radius $r_{0}=0.61 \lambda_{S} f_{1} / R_{1} \simeq 39 \mu \mathrm{m}$. As in practice, the vortex conversion efficiency is $\simeq 80 \%$, the LCLV is placed between crossed circular polarizers ( $C P$ and $C A)$. Starlight rejection is ensured by placing an iris $\left(I_{2}\right.$, also called the Lyot stop) with radius $R_{2}=0.75 R_{1} f_{2} / f_{1}$ in the exit pupil plane (whose location at a distance $f_{2}\left(1+f_{2} / f_{1}\right)$ from the second lens $\left(L_{2}\right)$ of the telescope is derived following the description provided by Collins based on the Fresnel diffraction integral [29]) where $R_{1} f_{2} / f_{1}$ is the radius of the disk of null intensity in the exit pupil plane in the case of an ideal amplitude transmission function $\tau_{\text {ideal }}[11]$.

The azimuth-averaged intensity distribution of the reimaging source $S_{1}$ (i.e., the focal plane of the lens $L_{4}$, see Fig. 2) when the coronagraph is $\mathrm{ON}$ (starlight and umbilic core are centered) and OFF (starlight is offset, but within the writing beam area) is plotted as a function of the reduced angular coordinate $\alpha / \alpha_{\text {diff }}$ where $\alpha_{\text {diff }}=0.61 \lambda_{S} / R_{1}$ is the diffraction limit, see Fig. 3. The experimental data (thick curves) are supported by simulations (thin curves) accounting for the transmission function of an umbilical vortex mask [24], $\tau(r, \phi)=\sin [\Delta(r) / 2] \exp [2 i \psi(r, \phi)+i \Delta(r) / 2]$, where the above formulations for $\Delta(r)$ and $\psi(r, \phi)$ are used. The images of $S_{1}$ when the coronagraph is OFF and ON are then calculated via a sequence of successive Fourier transforms [30] that describe the propagation of light from the input pupil plane to the camera, see Fig. 2. Namely, $I_{S} \propto$ $\left|\mathcal{F}\left[\operatorname{circ}\left(r / R_{2}\right) \mathcal{F}{ }^{1}\left[\tau \mathcal{F}\left[\operatorname{circ}\left(r / R_{1}\right)\right]\right]\right]\right|^{2}$ where $\mathcal{F}\left(\mathcal{F}^{1}\right)$ refers to the (inverse) Fourier transform and $\operatorname{circ}(x)=1$ for $x<1$ and zero otherwise.

As a writing beam can be steered on-demand, a given defect can be precisely pinned at any location. Therefore, by using $N$ independently steerable writing light beams $W_{i}$,

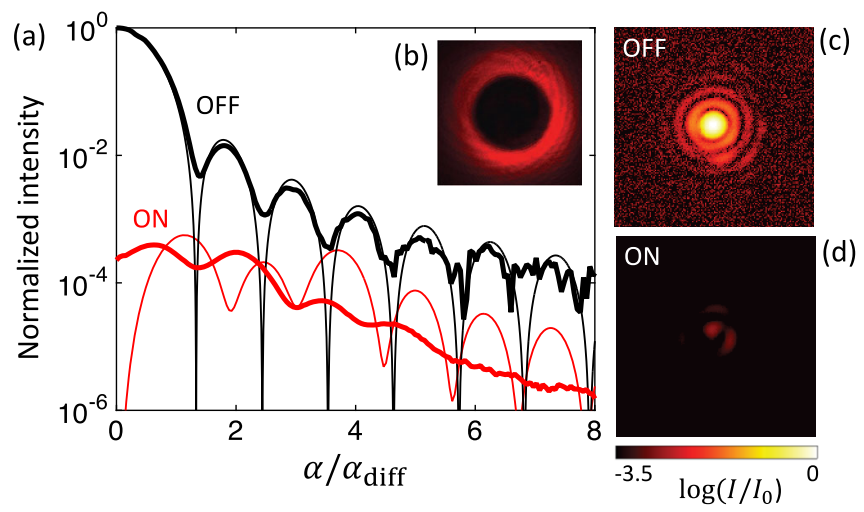

FIG. 3. (a) Azimuth averaged angular intensity distribution when the single star coronagraph is OFF (black curves) and ON (red curves) as a function of the reduced angular coordinate $\alpha / \alpha_{\text {diff }}$. Thick curves: experimental data; thin curves: simulations. (b) Ring of fire in the exit pupil plane. Experimental reimaged pointlike sources when the coronagraph is OFF and ON are shown on panels (c) and (d) with $I_{0}$ being the maximal intensity in the OFF state and where the logarithmic intensity range refers to the 4096 camera levels. The incident starlight is right handed circularly polarized.

$i=(1,2, \ldots, N)$, one can create $N$ vortex masks $M_{i}$ adapted to a system of $N$ stars $S_{i}$, as depicted in Fig. 2. This is illustrated by considering a triple-star system $(N=3)$, as shown in Fig. 4 where the Airy spots of the three stars in the focal plane of the telescope are shown in panel (a) when the LCLV is at rest (i.e., without applied electric field). The three writing beams are then positioned to overlap the defect core of each vortex mask when the electric field is applied, as shown in Fig. 4(b).

The obtained multiple-vortex mask is then used to perform an optical vortex coronagraphy laboratory demonstration of a system that consists of a triple star plus a planet. This is done by adding a faint off-axis illumination (the "planet," see $P$ in Fig. 2) at an angular separation of $\simeq 2 \alpha_{\text {diff }}$ from the star $S_{1}$. In practice, the values of the ratios between the maximal intensity of the stars $\left(S_{1}, S_{2}, S_{3}\right)$ and that of the planet are $(20.7: 19.4: 19.2)$, with an average total power for each star $P_{S_{i}} \simeq 13 \mu \mathrm{W}$ and the average total power for each writing beam is $P_{W_{i}} \simeq 0.39 \mathrm{~mW}$. We note that we checked that the starlight has no detectable influence on the vortex mask writing. Experimental data are displayed in Fig. 5 where the panel (a) refers to the OFF state of the coronagraph while the panel (b) refers to the ON state. The observations are compared with simulations taking the same conditions as in experiments, see in Figs. 5(c) and 5(d).

Regarding present results, there is of course some room for improvement. In particular, both the spontaneous broken axial and chiral symmetries should be improved, for instance, by alternative topological structuring strategies and the development of novel optical materials. The total transmission of the instrument is another aspect that should 

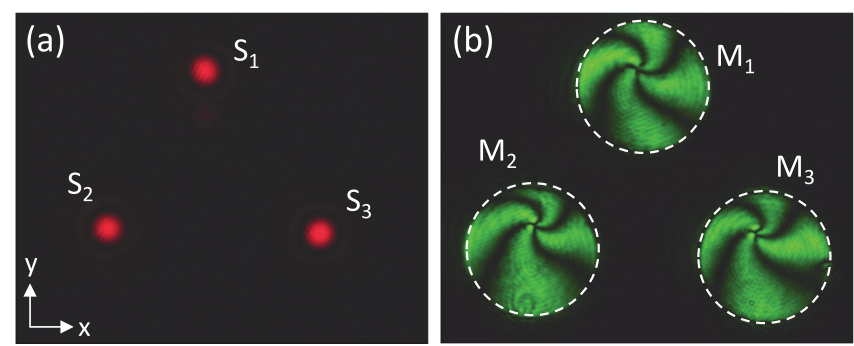

FIG. 4. (a) Airy spots of a triple star system $\left(S_{1}, S_{2}, S_{3}\right)$ in the focal plane of the telescope. (b) XPOL focal plane image of the corresponding vortex masks $\left(M_{1}, M_{2}, M_{3}\right)$ generated by the writing beams $\left(W_{1}, W_{2}, W_{3}\right)$ where the dashed circles indicate the contours of the illumination disks.

be optimized, especially by improving the purity of the generated vortex. In addition, the existence of dead zones for off-axis planet detection when carrying out observations between crossed circular polarizers and the possible interaction between two nearby vortex masks are two issues that also require further work. Finally, we note that the nonsingular nature of umbilical defects (i.e., the XCPOL

\section{Experiment}
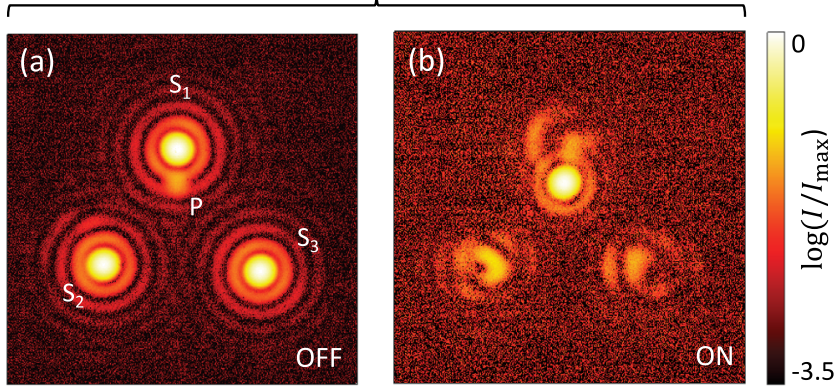

Model

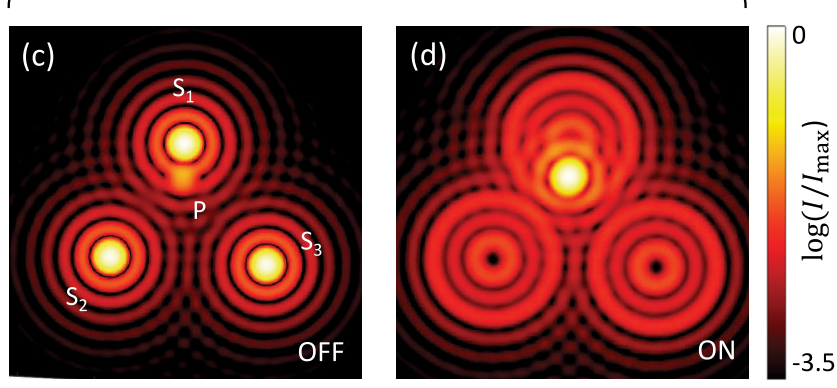

FIG. 5. Optical vortex coronagraphy laboratory demonstration of a system that consists of a triple star plus a planet. Reimaged light sources $\left(S_{1}, S_{2}, S_{3}\right)$ and planet $P$ with lens $L_{4}$ of focal length $f_{4}=500 \mathrm{~mm}$ when the coronagraph is OFF (a) and ON (b) with $I_{\max }$ being the maximal intensity of each image and where the logarithmic intensity range refers to the 4096 camera levels. Note that higher noise level in panel (b) results from limited exposure time of the camera, which limits the dynamic range of the image. The incident starlight is right handed circularly polarized. The simulated counterparts of panels (a) and (b) are shown on panels (c) and (d). transmission drops continuously to zero in the defect core area) allows self-adapted central apodization of each vortex mask. This is associated with a perfect unit charge topological structuring down to the central part of the defect, as discussed in Ref. [24], which is not the case with any artificial LC vectorial vortex coronagraphs reported so far that require additional central obscuration disks [12,31-33]. We also note that the use of starlight as the writing beam itself would be in vain in the present case due to unavoidable mismatch between the defect and the center of the illuminated area. Still, such an attractive self-eclipsing option has been recently discussed by taking advantage of the photoelastic phenomenon [34].

Summarizing, we have introduced the use of optical vortex coronagraphy to address the issue of direct observation of faint companions nearby multiple-star systems, which remains so far a challenge to astronomical observations. A proof-of-principle laboratory demonstration has been made on a simulated system that consists of a triple star plus a planet by using three independently steerable light beams enabling the generation of three independent vortex masks in a liquid crystal light valve. The proposed technique can be easily implemented on an arbitrary number of sources thus extending previous efforts to develop smart optical vortex coronagraphs [24]. We also note that the future of direct imaging of exoplanets will not rely on a single technique but instead will rely on the combination of complementary instrumental developments covering coronagraphy, adaptive optics, sensors, and image processing, in conjunction with the next-generation giant telescopes (e.g., Extremely Large Telescope, Giant Magellan Telescope, and Thirty-Meter Telescope) endowed with unprecedented resolution.

This study has been carried out with financial support from the French National Research Agency (ANR) in the frame of the Investments for the future Programme IdEx Bordeaux LAPHIA (ANR-10-IDEX-0302) and HYPERPHORB project (ANR-15-CE30-0018). A. A and N. K contributed equally to this work.

Note added.-Recently, we became aware of a work reporting on the direct imaging discovery of a Jovian exoplanet within a triple-star system [35] and of a work dealing with the laboratory demonstration of high-contrast coronagraphy of simulated binary-star systems [36] using a pair of multiple-quadrant transmission phase masks.

*etienne.brasselet@u bordeaux.fr

[1] M. Mayor and D. Queloz, A Jupiter mass companion to a solar type star, Nature (London) 378, 355 (1995).

[2] F. Pepe, D. Ehrenreich, and M. R. Meyer, Instrumentation for the detection and characterization of exoplanets, Nature (London) 513, 358 (2014).

[3] J. F. Kasting, D. P. Whitmire, and R. T. Reynolds, Habitable zones around main sequence stars, Icarus 101, 108 (1993). 
[4] M. Perryman, The Exoplanet Handbook (Cambridge University Press, Cambridge, England, 2014).

[5] G. Chauvin, A. M. Lagrange, C. Dumas, B. Zuckerman, D. Mouillet, I. Song, J. L. Beuzit, and P. Lowrance, Giant planet companion to 2massw j1207334 393254, Astron. Astrophys. 438, L25 (2005).

[6] D. Mawet, P. Riaud, O. Absil, and J. Surdej, Annular groove phase mask coronagraph, Astrophys. J. 633, 1191 (2005).

[7] G. Foo, D. M. Palacios, and G. A. Swartzlander, Optical vortex coronagraph, Opt. Lett. 30, 3308 (2005).

[8] B. Lyot, A study of the solar corona and prominences without eclipses, Mon. Not. R. Astron. Soc. 99, 580 (1939).

[9] P. Kalas, J. R. Graham, E. Chiang, M. P. Fitzgerald, M. Clampin, E. S. Kite, K. Stapelfeldt, C. Marois, and J. Krist, Optical images of an exosolar planet 25 light years from earth, Science 322, 1345 (2008).

[10] E. Serabyn, D. Mawet, and R. Burruss, An image of an exoplanet separated by two diffraction beamwidths from a star, Nature (London) 464, 1018 (2010).

[11] G. A. Swartzlander, The optical vortex coronagraph, J. Opt. A: Pure Appl. Opt. 11, 094022 (2009).

[12] O. Absil et al., Three years of harvest with the vector vortex coronagraph in the thermal infrared, Proc. SPIE Int. Soc. Opt. Eng. 9908, 99080Q (2016).

[13] The Extrasolar Planets Encyclopaedia, http://exoplanet.eu/ catalog/.

[14] R. Doyle et al., Kepler 16: A transiting circumbinary planet, Science 333, 1602 (2011).

[15] J. A. Orosz et al., Kepler 47: A transiting circumbinary multiplanet system, Science 337, 1511 (2012).

[16] J. Crepp, E. Serabyn, J. Carson, J. Ge, and I. Kravchenko, On ky demonstration of a linear band limited mask with application to visual binary stars, Astrophys. J. 715, 1533 (2010).

[17] E. Cady, M. McElwain, N. J. Kasdin, and C. Thalmann, A dual mask coronagraph for observing faint companions to binary stars, Publ. Astron. Soc. Pac. 123, 333 (2011).

[18] S. Thomas, R. Belikov, and E. Bendek, Techniques for high contrast imaging in multi star systems. I. Super Nyquist wavefront control, Astrophys. J. 810, 81 (2015).

[19] E. Brasselet, Spin orbit optical cross phase modulation, Phys. Rev. A 82, 063836 (2010).

[20] E. Brasselet and C. Loussert, Electrically controlled topo logical defects in liquid crystals as tunable spin orbit encoders for photons, Opt. Lett. 36, 719 (2011).

[21] R. Barboza, U. Bortolozzo, G. Assanto, E. Vidal Henriquez, M. G. Clerc, and S. Residori, Vortex Induction via Anisotropy Stabilized Light Matter Interaction, Phys. Rev. Lett. 109, 143901 (2012).

[22] I. A. Budagovsky, A. S. Zolotko, M. P. Smayev, and S. A. Shvetsov, Formation of the light beam with wavefront screw dislocation at the photorefractive effect in nematic liquid crystal, Bulletin of the Lebedev Physics Institute 42, 319 (2015).

[23] I. A. Budagovsky, A. S. Zolotko, D. L. Korshunov, M. P. Smayev, S. A. Shvetsov, and M. Barnik, Generation of spiral dislocation of wave front in absorbing nematic liquid crystal, Opt. Spectrosc. 119, 280 (2015).

[24] A. Aleksanyan and E. Brasselet, Vortex coronagraphy from self engineered liquid crystal spin orbit masks, Opt. Lett. 41, 5234 (2016).

[25] P. Aubourg, J. P. Huignard, M. Hareng, and R. A. Mullen, Liquid crystal light valve using bulk monocrystalline $\mathrm{Bi}_{12} \mathrm{SiO}_{20}$ as the photoconductive material, Appl. Opt. 21, 3706 (1982).

[26] R. Barboza, U. Bortolozzo, M. G. Clerc, S. Residori, and E. Vidal Henriquez, Light matter interaction induces a single positive vortex with swirling arms, Phil. Trans. R. Soc. A 372, 20140019 (2014).

[27] A. Rapini, Umbilics: Static properties and shear induced displacements, J. Phys. USSR 34, 629 (1973).

[28] E. Brasselet, Tunable Optical Vortex Arrays from a Single Nematic Topological Defect, Phys. Rev. Lett. 108, 087801 (2012).

[29] S. A. Collins, Lens system diffraction integral written in terms of matrix optics, J. Opt. Soc. Am. 60, 11681177 (1970).

[30] J. W. Goodman, Introduction to the Fourier optics (Roberts and Company Publishers, Englewood, CO, 2005).

[31] D. Mawet, E. Serabyn, K. Liewer, C. Hanot, S. McEldowney, D. Shemo, and N.E. O'Brien, Optical vectorial vortex coronagraphs using liquid crystal polymers: theory, manufacturing and laboratory demonstration, Opt. Express 17, 1902 (2009).

[32] D. Mawet, J. T. Trauger, E. Serabyn, D. C. Moody, K. M. Liewer, J. E. Krist, D. M. Shemo, and N. A. O'Brien, Vector vortex coronagraph: first results in the visible, Proc. SPIE Int. Soc. Opt. Eng. 7440, 74400X (2009).

[33] D. Mawet, E. Serabyn, K. Liewer, K. Burruss, J. Hickey, and D. Shemo, The vector vortex coronagraph: Laboratory results and first light at Palomar observatory, Astrophys. J. 709, 53 (2010).

[34] A. Aleksanyan and E. Brasselet, Self eclipsing: alignment free vortex coronagraphy, Opt. Lett. 42, 1237 (2017).

[35] K. Wagner, D. Apai, M. Kasper, K. Kratter, M. McClure, M. Robberto, and J. L. Beuzit, Direct imaging discovery of a Jovian exoplanet within a triple star system, Science 353, 673 (2016).

[36] J. Kühn and P. Patapis, Digital adaptive coronagraphy using SLMs: Promising prospects of a novel approach, including high contrast imaging of multiple stars sys tems, Proc. SPIE Int. Soc. Opt. Eng. 9912, 99122M (2016). 